\section{Dementia

\title{
Combining the Rowland Universal Dementia Assessment Scale and the Informant Questionnaire on Cognitive Decline in the Elderly to Improve Detection of Dementia in an Arabic-Speaking Population
}

\author{
T. Rune Nielsen ${ }^{a} \quad$ Thien Kieu Thi Phung $^{a} \quad$ Monique Chaaya ${ }^{b}$ \\ Andrew Mackinnon ${ }^{c}$ Gunhild Waldemar ${ }^{\mathrm{a}}$ \\ aDanish Dementia Research Centre, Rigshospitalet, University of Copenhagen, Copenhagen, \\ Denmark; ${ }^{b}$ Department of Epidemiology and Population Health, American University of \\ Beirut, Beirut, Lebanon; ${ }^{\mathrm{C} O r y g e n, ~ T h e ~ N a t i o n a l ~ C e n t r e ~ o f ~ E x c e l l e n c e ~ i n ~ Y o u t h ~ M e n t a l ~ H e a l t h, ~}$ \\ University of Melbourne, Melbourne, Vic., Australia
}

\section{Key Words}

Cognitive screening test - Dementia - Diagnostic accuracy · Arabic $\cdot$ Illiteracy

\begin{abstract}
Background/Aims: The aim of this study was to assess whether combining the Rowland Universal Dementia Assessment Scale (RUDAS) and Informant Questionnaire on Cognitive Decline in the Elderly (IQCODE) could improve diagnostic accuracy when screening for dementia in an Arabic-speaking population with low levels of literacy. Methods: Based on data from 225 elderly participant and informant dyads, the screening performances of the two instruments were compared and three methods for combining them - 'or', 'and' and 'weighted sum' rules - were assessed. Results: The 'weighted sum' method had a significantly better area under the curve value compared to the RUDAS used alone. The 'weighted sum' method and the 'and' rule had the highest specificity, while the 'or' rule had the best sensitivity. Conclusion: Harnessing the RUDAS and IQCODE increased diagnostic accuracy when screening for dementia in this study population.

(C) 2015 The Author(s)

Published by S. Karger AG, Basel
\end{abstract}

\section{Introduction}

In North Africa and the Middle East (WHO Eastern Mediterranean Region or EMRO), the population is rapidly aging at an unprecedented rate [1]. The number of people with dementia is expected to increase from 1.2 million people in 2010 to 2.6 million in 2030 and to 6.2 
Dementia

and Geriatric

Cognitive Disorders \begin{tabular}{l|l}
\hline Dement Geriatr Cogn Disord 2016;41:46-54 \\
\hline DOI: 10.1159/000441649 & $\begin{array}{l}\text { ( ) 2015 The Author(s). Published by S. Karger AG, Basel } \\
\text { www.karger.com/dem }\end{array}$ \\
\hline
\end{tabular}

Nielsen et al.: Combining the RUDAS and the IQCODE to Improve Detection of

Dementia in an Arabic-Speaking Population

million in 2050, the second fastest rate of increase in the world [2]. Clearly, timely diagnosis and interventions for people with dementia as well as psychosocial support for their family caregivers are becoming increasingly important in the region.

Accurate diagnoses are required prior to any intervention, and cognitive assessment is the cornerstone of the diagnostic process for dementia. However, the high rate of illiteracy among elderly people in the EMRO renders cognitive assessment very challenging, since commonly used cognitive screening tests, such as the Mini-Mental State Examination (MMSE) [3], require reading, writing and arithmetic skills. We have previously validated the Rowland Universal Dementia Assessment Scale (RUDAS) [4], a brief cognitive screening instrument that was specifically designed to minimize the effect of culture, language and education, and also the Informant Questionnaire on Cognitive Decline in the Elderly (IQCODE) and have shown that both possessed good screening performance for dementia in an Arabic-speaking older population with little education $[5,6]$. In particular the psychometric properties of the IQCODE were found to be excellent. Although the RUDAS was designed to minimize the effect of education, we found test performances to be influenced by education in this population, in particular among those with no formal education. However, this did not affect the diagnostic accuracy of the Arabic RUDAS (A-RUDAS) when adopting the recommended cutoff value [5]. In contrast, we found no influence of education on the IQCODE [6], a result consistent with previous studies of the RUDAS and IQCODE in older populations from low- and middleincome (LMIC) countries with little education [7-12].

The great challenge of cognitive testing of illiterate elderly people underlines the importance of supporting the findings from cognitive testing with information from a knowledgeable informant to reduce educational test bias [13]. Supplementing brief cognitive screening instruments such as the MMSE with informant reports based on structured questionnaires such as the IQCODE has been found to improve diagnostic accuracy when screening for dementia [14-16], in particular in populations with a high proportion of elderly with little or no formal education [15]. Three different methods of combining the MMSE with the IQCODE have been suggested, namely the 'and' rule, the 'or' rule and the 'weighted sum' method [16]. The RUDAS and MMSE are conceptually similar instruments that are highly correlated in clinical settings, with correlation coefficients in the range of $0.73-0.85$ [8, 11, 17-21]. This suggests that the same approach might be used to combine the A-RUDAS and the IQCODE.

The aim of this study was to assess whether combining the A-RUDAS and IQCODE could improve diagnostic accuracy when screening for dementia in an Arabic-speaking population with a high rate of illiteracy. Data for the study were derived from a validation study for three cognitive assessments in Arabic using the same study population: (1) the 10/66 Dementia Research Group (DRG) one-phase diagnostic assessment [22], (2) the A-RUDAS and (3) the IQCODE.

\section{Subjects and Methods}

Data collection was completed from March 2012 to February 2013. Phung et al. [22] provide specific details on the study. For the purposes of the present study, only data from elderly participants and informant dyads with both A-RUDAS and IQCODE data available were analyzed.

Participants

Elderly participants enrolled in the study were from the communities, social organizations for the elderly, community-based primary care clinics as well as hospital-based geriatric and neurological clinics. Inclusion criteria were (1) age of 65 years and older, (2) having normal cognition (controls) or mild-tomoderate dementia (cases) and (3) having a knowledgeable informant who could give an independent assessment of the elderly participant's health and cognitive status. Exclusion criteria were (1) severe somatic 
Nielsen et al.: Combining the RUDAS and the IQCODE to Improve Detection of

Dementia in an Arabic-Speaking Population

or psychiatric illness, when the diagnosis of dementia could not be ascertained without extensive medical workup and neuropsychological testing, and (2) mild cognitive impairment (MCI) diagnosed according to the core clinical criteria for MCI by the National Institute on Aging-Alzheimer's Association (NIA-AA) workgroups [23].

Informants were identified as the persons who were most involved in the care of the elderly participants or most knowledgeable about the mental and health status of the participants through regular contacts over a time frame of at least 1 year. The informants could therefore be lay persons (relatives, friends or social workers) or health professionals (nurses or physiotherapists). They were mainly family caregivers for the elderly participants living in the community, and mainly the nurses in charge of the older participants residing in nursing homes.

A total of 244 elderly participant and informant dyads were recruited, but only 225 were eligible for inclusion in the present study; 11 were excluded due to missing data on the A-RUDAS, 7 due to missing items on the IQCODE, and 1 due to missing data on both. Of the 225 included participant and informant dyads, 90 comprised dementia patients, 48 mild dementia patients [Clinical Dementia Rating (CDR) = 1] and 42 moderate dementia patients $(\mathrm{CDR}=2)$.

The study was approved by the Institutional Review Board of the American University of Beirut. All participants gave written informed consent. In case a participant had impaired decision-making, the primary caregiver gave consent on his/her behalf.

\section{Dementia Screening Instruments}

The RUDAS [4] is a brief screening instrument that was developed as an alternative to the MMSE to screen for cognitive impairment in multicultural populations. The RUDAS contains 6 items, namely body orientation, praxis, drawing, judgment, memory and language. Like the MMSE, the RUDAS has a score interval of $0-30$ points, is easy to administer and takes about $10 \mathrm{~min}$ to complete. It has been validated in several clinical and cultural contexts [4, 7, 10-12,17, 18, 20,21] including Arabic [5], generally showing good psychometric properties that are comparable or slightly superior to the commonly used MMSE.

The IQCODE is a brief screening assessment for cognitive impairment based on an interview with an informant, usually a family caregiver [24]. The short form of the IQCODE contains 16 questions and rates changes in cognitive functioning of an older adult over a specified time frame. Each question of the IQCODE is rated on a 5-point scale from 1 (much improved) to 5 (much worse) with 3 representing no change. The sum of scores is averaged over the 16 items to give a score of $1-5$. Higher scores indicate greater impairment. For the short form of the IQCODE to be valid, a maximum of 2 missing items are allowed [24]. The IQCODE has been validated in different languages and cultures as well as in populations with low education, showing psychometric properties comparable to those of the MMSE [9].

All assessments were performed by health care professionals ( 3 neurological fellows, 1 geriatric fellow and 1 family medicine resident) and 1 non-medical university graduate (Master of Public Health), all of whom were trained and supervised in administering the RUDAS and IQCODE. The interviewers, who were blinded to the cognitive status of the participants, administered the RUDAS to the elderly participants and the IQCODE to the informants.

\section{Diagnoses}

Dementia and MCI diagnoses among the elderly participants were established by a consensus between clinicians according to the Diagnostic and Statistical Manual of Mental Disorders (DSM-IV) [25] and NIA-AA [23] criteria based on all available clinical information, excluding results from the RUDAS and IQCODE. In case the available clinical information was insufficient to establish a diagnosis, additional cognitive assessments of the participants and interviews with caregivers were carried out. Dementia severity was rated using the CDR [26]. The mental status of the elderly participants was assessed with the Geriatric Mental State (GMS) [27], and depression was diagnosed using GMS/AGECAT stage 1 output, which has a sensitivity of $90 \%$ for diagnosing depression among elderly people with little education in LMIC [28].

\section{Statistical Analyses}

Group comparisons were made using a number of parametric and nonparametric statistical tests. Differences between categorical variables were tested with the Pearson $\chi^{2}$ test or Fisher's exact test, when appropriate. Differences between continuous variables were tested with analyses of variance, or the MannWhitney test when the assumptions of parametric testing were not fulfilled. Spearman's $\rho$ was used to assess associations between continuous variables. 
Nielsen et al.: Combining the RUDAS and the IQCODE to Improve Detection of Dementia in an Arabic-Speaking Population

Table 1. Demographic and clinical characteristics

\begin{tabular}{lcc}
\hline Characteristic & Dementia patients $(\mathrm{n}=90)$ & Controls (n=135) \\
\hline Mean age \pm SD, years*** & $81.9 \pm 7.5$ & $77.0 \pm 7.5$ \\
Female gender & $61(67.8)$ & $83(61.5)$ \\
Education* & $55(61.1)$ & $61(45.2)$ \\
$\quad$ No formal education & $16(17.8)$ & $23(17.0)$ \\
$\quad$ Primary education & $11(12.2)$ & $19(14.1)$ \\
$\quad$ Intermediate education & $7(7.8)$ & $18(13.3)$ \\
$\quad$ Secondary education & $1(1.1)$ & $69(10.4)$ \\
$\quad$ University and above & $44(48.9)$ & $26(24-27)$ \\
Depression & $17(12-21.5)$ & $3(3-3.13)$ \\
Median RUDAS score (Q1-Q3) & $4.16(3.66-4.69)$ & \\
Median IQCODE score (Q1-Q3) & (2** &
\end{tabular}

Values are presented as n (\%) unless specified otherwise. Q1-Q3 = 1st quartile to 3rd quartile. * $\mathrm{p}<0.05$; $* * * \mathrm{p}<0.001$.

Logistic regression analyses were used to determine whether the two instruments considered together provided any additional information for predicting dementia over any of the tests used alone. In two models predicting DSM-IV dementia caseness, either the RUDAS or the IQCODE score was used as the basis for the equation to confirm that each instrument yielded nonredundant information in the prediction of dementia. Quadratic and multiplicative terms were added to the models to assess the possibility of nonlinear relationships between dementia caseness and test scores as well as possible interactions between the instruments.

Combinations of the RUDAS and IQCODE were evaluated using the 'or' rule (either the RUDAS or the IQCODE in the impaired range), the 'and' rule (both the RUDAS and the IQCODE in the impaired range) or the 'weighted sum' method (probability of a dementia case $>0.5$ from the logistic regression equation). These definitions were consistent with previous methods used to combine the MMSE and the IQCODE [14-16]. Test performance was evaluated using receiver operating characteristic (ROC) curve analyses. The areas under the ROC curve (AUC) were used to compare the overall discriminatory power of the RUDAS, the IQCODE and the combined measures in differentiating between people with dementia and cognitively intact controls.

In previous validation studies in the same population, we established the optimal cutoff values to be $<23 / 30$ for the RUDAS [5] and $>3.34 / 5$ for the IQCODE [6]. These cutoff values were used for the 'and' and 'or' analyses. Sensitivity, specificity, AUC, positive predictive values (PPV), negative predictive values (NPV), positive likelihood ratios (LR+) and negative likelihood ratios (LR-) were evaluated for the individual screening instruments and the combined measures.

Clinical calculators from the VassarStats website were used to calculate the sensitivity, specificity, PPV, NPV, LR+ and LR- with 95\% CI (www.vassarstats.net/clin1.html) and to test for significant differences between AUC (vasserstats.net/roc_comp.html). All other analyses were performed with SPSS statistical software (version 19.0; SPSS Inc., Chicago, Ill., USA). p < 0.05 (two-tailed) was considered significant.

\section{Results}

The demographic and clinical characteristics of the study population are presented in table 1. Compared to healthy controls, patients with dementia were older $[\mathrm{F}(1,223)=21.35$, $\mathrm{p}<0.001]$ and less educated $\left[\chi^{2}(5, \mathrm{n}=225)=13.65, \mathrm{p}=0.018\right]$. No differences were found in the distribution of genders (Fisher's exact test, $\mathrm{p}=0.395$ ) or the presence of depression (Fisher's exact test, $\mathrm{p}=0.786$ ). As expected, patients with dementia had significantly lower scores on the RUDAS $(U=939, \mathrm{p}<0.001)$ and significantly higher scores on the IQCODE $(U=$ $11,755, \mathrm{p}<0.001$ ). RUDAS and IQCODE scores where substantially correlated (Spearman's $\rho=-0.66, \mathrm{p}<0.001)$. 
Dementia

Cognitive Disorders

Table 2. Logistic regression analysis for dementia diagnosis $(n=225)$

\begin{tabular}{l|l}
\hline Dement Geriatr Cogn Disord 2016;41:46-54 \\
\hline DOI: 10.1159/000441649 & $\begin{array}{l}\text { @ 2015 The Author(s). Published by S. Karger AG, Basel } \\
\text { www.karger.com/dem }\end{array}$ \\
\hline
\end{tabular}

Nielsen et al.: Combining the RUDAS and the IQCODE to Improve Detection of Dementia in an Arabic-Speaking Population

\begin{tabular}{lrr}
\hline & Change in log likelihood & $\mathrm{p}$ \\
\hline Model 1 & & \\
$\quad$ RUDAS & 148.1 & $<0.001$ \\
RUDAS $^{2}$ & 0.001 & 0.970 \\
IQCODE & 79.5 & $<0.001$ \\
RUDAS $\times$ IQCODE & 1.4 & 0.080 \\
Model 2 & & \\
IQCODE & 208.3 & $<0.001$ \\
IQCODE $^{2}$ & 0.02 & 0.875 \\
RUDAS & 19.1 & $<0.001$ \\
RUDAS $\times$ IQCODE & 0.8 & 0.223 \\
\hline
\end{tabular}

Table 3. Diagnostic accuracy of the RUDAS, IQCODE and combined measures $(n=225)$

\begin{tabular}{|c|c|c|c|c|c|c|c|}
\hline Measure & AUC & Sensitivity (95\% CI) & Specificity (95\% CI) & PPV (95\% CI) & NPV (95\% CI) & $\mathrm{LR}+(95 \% \mathrm{CI})$ & LR- $(95 \% \mathrm{CI})$ \\
\hline RUDAS & 0.923 & $0.82(0.72-0.89)$ & $0.84(0.77-0.90)$ & $0.78(0.68-0.93)$ & $0.88(0.8$ & $5.29(3.53-7.92)$ & $0.21(0.13-0.33)$ \\
\hline IQCODE & 0.967 & $0.92(0.84-0.96)$ & $0.96(0.90-0.98)$ & $0.93(0.85-0.97)$ & $0.95(0.89-0.98)$ & $20.75(9.47-45.47)$ & $0.08(0.04-0.17)$ \\
\hline 'Or' rule & 0.900 & $0.96(0.88-0.99)$ & $0.84(0.77-0.90)$ & $0.80(0.71-0.87)$ & $0.97(0.91-0.99)$ & $6.14(4.14-9.12)$ & $0.05(0.02-0.14)$ \\
\hline 'And' rule & 0.863 & $0.76(0.65-0.84)$ & $0.97(0.92-0.99)$ & $0.94(0.86-0.98)$ & $0.86(0.79-0.91)$ & $25.5(9.64-67.43)$ & $0.25(0.18-0.36)$ \\
\hline 'Weighted sum' method & 0.974 & $0.86(0.76-0.92)$ & $0.97(0.92-0.99)$ & $0.95(0.87-0.98)$ & $0.91(0.85-0.95)$ & $28.88(10.96-76.10)$ & $0.15(0.09-0.25)$ \\
\hline
\end{tabular}

Weighted Combination of the RUDAS and the IQCODE

The results of the logistic regression analyses performed to obtain the 'weighted sum' equation are summarized in table 2. The larger the value for the change in log likelihood, the greater the importance of a test, or combination, is to the accuracy of predictions. Adding the RUDAS or IQCODE to models containing the other test resulted in highly statistically significant improvements in prediction of caseness in both models. Inclusion of quadratic terms and the interaction between the RUDAS and IQCODE were not included in the final regression equation, as they did not have any significant effect on the change in log likelihood. Thus, it can be concluded that each test provides unique information on the prediction of dementia and that the relationship between the logit of caseness and test scores is essentially linear. The equation derived from the logistic regression for predicting the likelihood of meeting caseness criteria for dementia for a given RUDAS and IQCODE score combination is shown below:

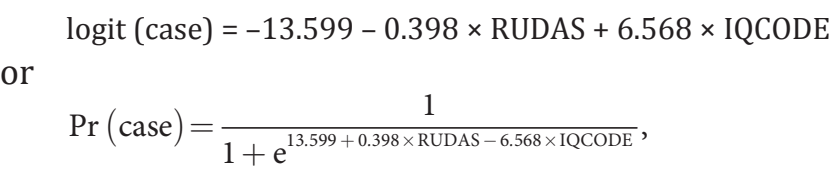

where 'logit (case)' is the algorithm of the odds of caseness, which is the natural log (probability of caseness/probability of non-caseness). 'Pr (case)' is the probability of caseness. RUDAS and IQCODE are the test score values.

\section{Screening Performance of the Combined Measures}

The ROC for the RUDAS, IQCODE, 'or' rule, 'and' rule and 'weighted sum' method are presented in figure 1. Table 3 summarizes the psychometric properties of the RUDAS, the IQCODE and the different methods for combining the instruments. Used alone, the IQCODE outperformed the RUDAS in all comparisons. The 'weighted sum' method and the 'and' rule had the best specificity, PPV and LR+, while the 'or' rule had the best sensitivity, NPV and LR-. 
Fig. 1. ROC curves for the RUDAS, IQCODE and combined measures

Nielsen et al.: Combining the RUDAS and the IQCODE to Improve Detection of Dementia in an Arabic-Speaking Population

for detecting dementia $(n=225)$.

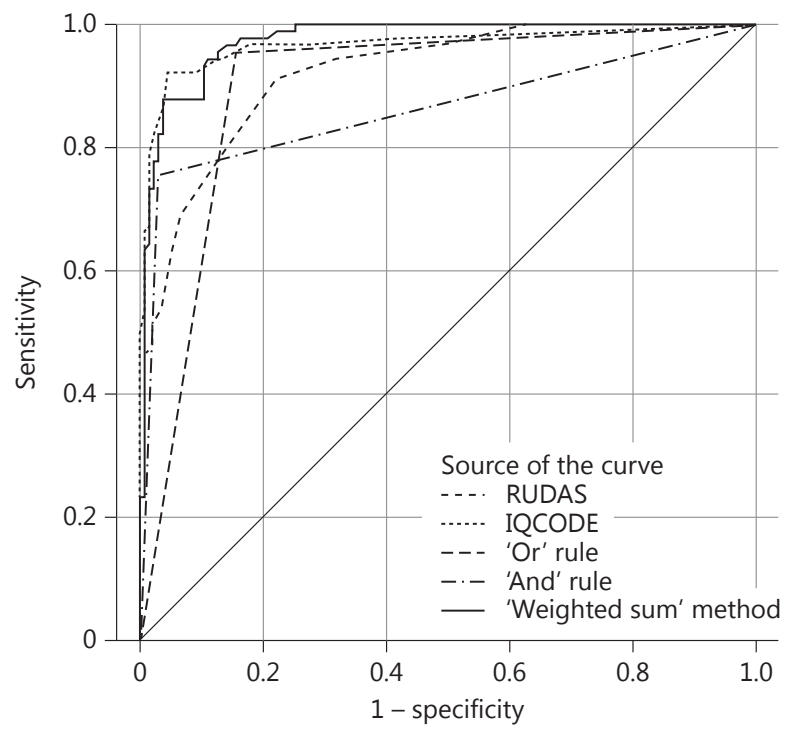

The 'weighted sum' method resulted in the highest AUC value, and was the only AUC value that was significantly different from the AUC value for RUDAS used alone $(\mathrm{z}=-2.47, \mathrm{p}=$ $0.014)$. There was no significant difference between the AUC for the RUDAS and IQCODE $(\mathrm{z}=-1.352, \mathrm{p}=0.176)$.

\section{Discussion}

This is the first study to combine cognitive testing with the RUDAS with informant reports from the IQCODE. In our study population of Arabic-speaking elderly participants, half of whom were illiterate, combining the RUDAS and IQCODE generally performed very well in screening for dementia. When a high specificity is desired, the 'weighted sum' method seems preferable, while the 'or' rule seems preferable when a high sensitivity is desired.

Strengths of this study include that data collection was strictly standardized according to the procedures of the 10/66 DRG protocol through rigorous translation processes, training and supervision of assessors, and case ascertainment. Case ascertainment was performed by clinicians from teaching hospitals in Lebanon using well-validated clinical criteria. However, the quality of dementia diagnoses is a well-known methodological issue in population-based dementia research in LMIC [29]. A limitation of the data set is that some MCI or very mild dementia cases could be present among our controls because in-depth neuropsychological testing and ancillary investigations were not available. At the same time, however, this may also have led to some very mild dementia cases being excluded from the patient sample. That, together with the inclusion of participants from the long-term care setting, where the prevalence of dementia was higher and informants were mostly health care professionals, and the use of highly trained physicians as interviewers, could lead to an overestimation of the screening ability of the RUDAS and IQCODE.

Remarkably, combining the RUDAS and IQCODE with the 'weighted sum' method resulted in psychometric properties that were comparable to the 10/66 DRG diagnostic assessment 
Dementia

and Geriatric \begin{tabular}{l|l}
\hline Dement Geriatr Cogn Disord 2016;41:46-54 \\
\hline DOI: 10.1159/000441649 & $\begin{array}{l}\text { C } 2015 \text { The Author(s). Published by S. Karger AG, Basel } \\
\text { www.karger.com/dem }\end{array}$ \\
\hline
\end{tabular}

Nielsen et al.: Combining the RUDAS and the IQCODE to Improve Detection of Dementia in an Arabic-Speaking Population

in the same study population, both having AUC of 0.97 [22]. The 10/66 DRG diagnostic assessment also combines cognitive testing with a structured informant interview, but it is much more time-consuming. The time to complete the RUDAS and IQCODE when serially presented was just 20-30 min in comparison to 45-60 min for the 10/66 DRG diagnostic assessment [22]. This indicates that combining the RUDAS and IQCODE with the 'weighted sum' method has the potential for being a time-effective method of screening for cognitive impairment in epidemiological studies in LMIC. However, since the research workers in the study were physicians and a university graduate who underwent rigorous training and were closely supervised throughout their fieldwork, the same excellent results may not be found in less optimal conditions or in a clinical setting. Importantly, the 10/66 DRG diagnostic assessment can effectively be performed by trained lay graduate assessors without any clinical expertise [30], which may not be true for the RUDAS.

Consistent with results from previous studies combining the MMSE and IQCODE [14-16], combining the RUDAS and IQCODE with the 'weighted sum' method resulted in the best AUC value, specificity, PPV and LR+, while the 'or' rule had the best sensitivity, NPV and LR-. The IQCODE outperformed the RUDAS in all comparisons. Also, the 'weighted sum' method, while significantly better than the RUDAS when used alone, was not superior to the IQCODE. Similar results have been described in a study on combining the MMSE and IQCODE in Singapore [15], which contrasts the findings from Western study populations, where cognitive testing with instruments such as the MMSE and RUDAS is typically superior to informant report from the IQCODE in detecting dementia [17]. Narasimhalu et al. [15] suggested that this difference may be explained by the extended family structure within which most elderly individuals live in Singapore, which may have led to informant reports being more accurate than in Western populations, where the elderly often live independently. The same line of argumentation may be used to explain our findings, as most elderly subjects in Lebanon also live within extended family structures. However, it must be noted that in our validation study of the IQCODE, we found that health care professionals were better than family carers in detecting and reporting symptoms of cognitive decline, and they were less likely to misinterpret depression symptoms in the older persons as cognitive symptoms. Since about $40 \%$ of the informants were health care professionals, the excellent results from this validation study might not be reproduced in a real screening scenario, where informants are mostly lay persons [6]. Previous studies have shown that the IQCODE is affected by informant characteristics such as depression and anxiety in the informant, the level of a carer's burden and the quality of the relationship between the informant and the older persons they care for [24, 31]. Therefore, it is recommended that the IQCODE is combined with a cognitive test, such as the RUDAS, to improve screening accuracy.

In conclusion, our results suggest that, in an Arabic-speaking population with a high rate of illiteracy, it is valuable to combine the RUDAS and the IQCODE with the 'weighted sum' method, or to supplement the RUDAS with the IQCODE to increase diagnostic accuracy. Both instruments are freely available, and combining them seems to be a time- and costeffective way of improving the accuracy of screening for dementia in LMIC with a high proportion of elderly with little or no education. The regression analyses used for the 'weighted sum' method resulted in a linear expression, where only the RUDAS and the IQCODE were significant predictors of dementia. Thus, it is possible to construct a graphical template similar to the DemeGraph [16] to facilitate the 'weighted sum' method in clinical practice (fig. 2). This template, as well as a numerical method of inserting the RUDAS and IQCODE scores to obtain the 'weighted sum' probability of dementia, is available on request from the main author. 
Fig. 2. DemeGraph for combining cognitive testing with the RUDAS with informant report from the IQCODE. Scores from the RUDAS and IQCODE can be plotted on the DemeGraph template as an aid to assessing patients for dementia. Patients whose score profile is above the diagonal line (the unshaded portion of the graph) are likely to meet the criteria for dementia. Patients who fall below the line (the shaded part of the graph) are unlikely to meet the criteria for dementia.

\begin{tabular}{l|l}
\hline Dement Geriatr Cogn Disord 2016;41:46-54 \\
\hline DOI: 10.1159/000441649 & $\begin{array}{l}\text { C } 2015 \text { The Author(s). Published by S. Karger AG, Basel } \\
\text { www.karger.com/dem }\end{array}$ \\
\hline
\end{tabular}

Nielsen et al.: Combining the RUDAS and the IQCODE to Improve Detection of Dementia in an Arabic-Speaking Population

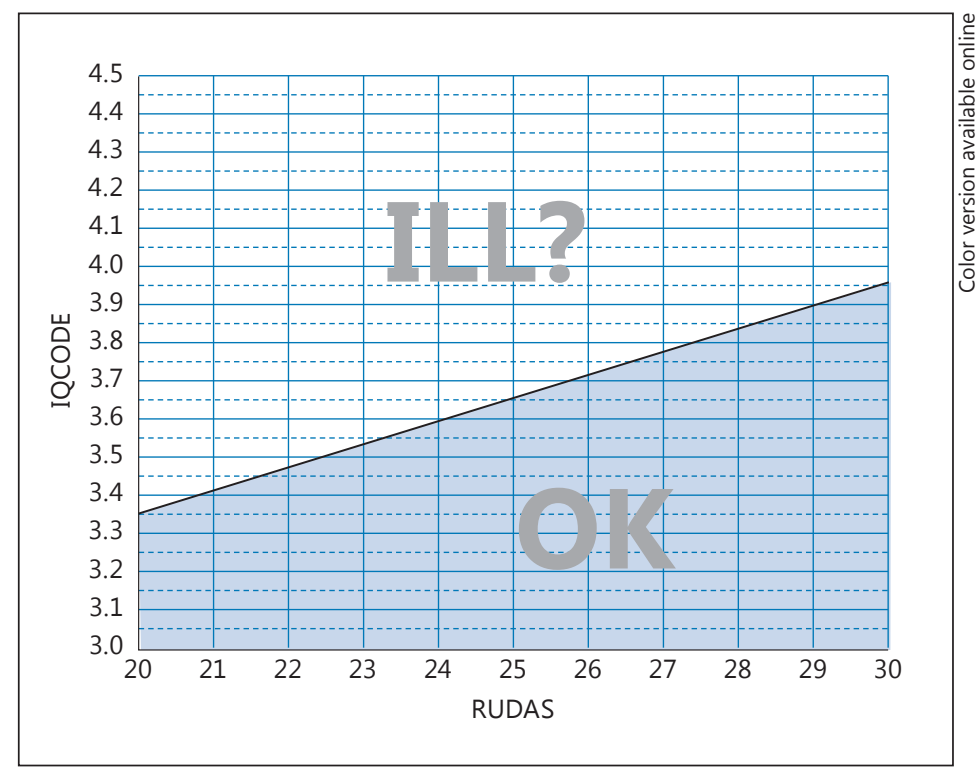

\section{Acknowledgements}

The data analyses and drafting of the paper were conducted as part of the Migrationsskole project under the EU-funded program Interreg IV A. The Danish Dementia Research Centre is supported by the Health Insurance Foundation and the Danish Ministry of Health and Prevention.

The fieldwork was funded by the Fogarty International Center, American National Institute of Health and National Institute of Aging [grant No. 1R21AG039333-01 under the program 'Brain Disorders in the Developing World: Research Across Lifespan (BRAIN)']. The content is solely the responsibility of the authors and does not necessarily represent the official views of the funding agencies. The recruitment was a community effort with contribution from institutions, organizations and individuals. Above all, the research group would like to express its deep gratitude to the participants in the study. We would also like to thank the Ministry of Social Affairs' Social Center in Bourj Hammoud; the NGO Restaurant du Coeur, the ladies from the Board of the Child and Mother Welfare Society and the Ayadina Social Center for the Elderly; Ain W. Zein, Hospital Geriatric Clinic; the Neurology Clinic at the American University of Beirut Medical Center; the geriatric clinics of Al Saydeh Hospital in Antelias, the Child and Mother Welfare Society and Baytouna Nursing Home; the primary care clinics of Maronite Nursing Home, Al Saydeh Church in Hamra and Al Salib Church in Naaba; the social workers Glady Farrah and Mona Saliby; Mrs. Dora Chaaya; Dean Iman Nuwayhid and Prof. Jihad Makhoul from the Faculty of Health Sciences, American University of Beirut, and Mrs. Helen Samaha, President of the Lebanese Order of Nursing. We thank Dr. Hussam Ghusn, Dr. Raja Sawaya and Dr. Nazem Bassil for their assistance with recruitment, and Mrs. Rose Mary Khoury, Dr. Ibrahim Zeinaty, Dr. Sandrine Salman, Dr. Salem Hammoud and Dr. Wael Radwan for their work as interviewers in the study. We thank the Alzheimer Association Lebanon for providing the educational materials about dementia for the patients and their caregivers in this study.

\section{References}

1 Sibai AM, Sen K, Baydoun M, Saxena P: Population ageing in Lebanon: current status, future prospects and implications for policy. Bull World Health Organ 2004;82:219-225.

2 Prince M, Bryce R, Albanese E, Wimo A, Ribeiro W, Ferri CP: The global prevalence of dementia: a systematic review and metaanalysis. Alzheimers Dement 2013;9:63-75.e2.

3 Folstein MF, Folstein SE, McHugh PR: 'Mini-mental state'. A practical method for grading the cognitive state of patients for the clinician. J Psychiatr Res 1975;12:189-198.

4 Storey JE, Rowland JT, Basic D, Conforti DA, Dickson HG: The Rowland Universal Dementia Assessment Scale (RUDAS): a multicultural cognitive assessment scale. Int Psychogeriatr 2004;16:13-31. 
5 Chaaya M, Phung TK, El Asmar K, Atweh S, Ghusn H, Khoury RM, Prince M, Nielsen TR, Waldemar G: Validation of the Arabic Rowland Universal Dementia Assessment Scale (A-RUDAS) in elderly with mild and moderate dementia. Aging Ment Health 2015, Epub ahead of print.

6 Phung TK, El Asmar K, Atweh S, Ghusn H, Khoury RM, Prince M, Waldemar G: Performance of the 16-item Informant Questionnaire on Cognitive Decline for the Elderly in an Arabic-speaking older population with low education. Alzheimers Dement 2014;10:P378.

7 Nielsen TR, Vogel A, Gade A, Waldemar G: Cognitive testing in non-demented Turkish immigrants - comparison of the RUDAS and the MMSE. Scand J Psychol 2012;53:455-460.

8 Naqvi RM, Haider S, Tomlinson G, Alibhai S: Cognitive assessments in multicultural populations using the Rowland Universal Dementia Assessment Scale: a systematic review and meta-analysis. CMAJ 2015;187:E169-E175.

9 Lin JS, O'Connor E, Rossom RC, Perdue LA, Eckstrom E: Screening for cognitive impairment in older adults: a systematic review for the US Preventive Services Task Force. Ann Intern Med 2013;159:601-612.

10 Iype T, Ajitha BK, Antony P, Ajeeth NB, Job S, Shaji KS: Usefulness of the Rowland Universal Dementia Assessment scale in South India. J Neurol Neurosurg Psychiatry 2006;77:513-514.

11 Limpawattana P, Tiamkao S, Sawanyawisuth K, Thinkhamrop B: Can Rowland Universal Dementia Assessment Scale (RUDAS) replace Mini-Mental State Examination (MMSE) for dementia screening in a Thai geriatric outpatient setting? Am J Alzheimers Dis Other Demen 2012;27:254-259.

12 Limpawattana P, Tiamkao S, Sawanyawisuth K: The performance of the Rowland Universal Dementia Assessment Scale (RUDAS) for cognitive screening in a geriatric outpatient setting. Aging Clin Exp Res 2012; 24:495-500.

13 Iype T, Ajitha BK, Shaji KS: Towards education-fair dementia screening. Int Psychogeriatr 2006;18:757-758.

14 Knafelc R, Lo Giudice D, Harrigan S, Cook R, Flicker L, Mackinnon A, Ames D: The combination of cognitive testing and an informant questionnaire in screening for dementia. Age Ageing 2003;32:541-547.

15 Narasimhalu K, Lee J, Auchus AP, Chen CP: Improving detection of dementia in Asian patients with low education: combining the Mini-Mental State Examination and the Informant Questionnaire on Cognitive Decline in the Elderly. Dement Geriatr Cogn Disord 2008;25:17-22.

16 Mackinnon A, Mulligan R: Combining cognitive testing and informant report to increase accuracy in screening for dementia. Am J Psychiatry 1998;155:1529-1535.

17 Goncalves DC, Arnold E, Appadurai K, Byrne GJ: Case finding in dementia: comparative utility of three brief instruments in the memory clinic setting. Int Psychogeriatr 2011;23:788-796.

18 Pang J, Yu H, Pearson K, Lynch P, Fong C: Comparison of the MMSE and RUDAS cognitive screening tools in an elderly inpatient population in everyday clinical use. Intern Med J 2009;39:411-414.

19 Basic D, Rowland JT, Conforti DA, Vrantsidis F, Hill K, Lo Giudice D, Harry J, Lucero K, Prowse RJ: The validity of the Rowland Universal Dementia Assessment Scale (RUDAS) in a multicultural cohort of communitydwelling older persons with early dementia. Alzheimer Dis Assoc Disord 2009;23:124-129.

20 Rowland JT, Basic D, Storey JE, Conforti DA: The Rowland Universal Dementia Assessment Scale (RUDAS) and the Folstein MMSE in a multicultural cohort of elderly persons. Int Psychogeriatr 2006;18:111-120.

21 Nielsen TR, Andersen BB, Gottrup H, Lützhøft JH, Høgh P, Waldemar G: Validation of the Rowland Universal Dementia Assessment Scale for multicultural screening in Danish memory clinics. Dement Geriatr Cogn Disord 2013;36:354-362.

22 Phung KT, Chaaya M, Waldemar G, Atweh S, Asmar K, Ghusn H, Karam G, Sawaya R, Khoury RM, Zeinaty I, Salman S, Hammoud S, Radwan W, Bassil N, Prince M: Validation of the 10/66 Dementia Research Group diagnostic assessment for dementia in Arabic: a study in Lebanon. J Geriatr Psychiatry Neurol 2014;27:282-290.

23 Albert MS, DeKosky ST, Dickson D, Dubois B, Feldman HH, Fox NC, Gamst A, Holtzman DM, Jagust WJ, Petersen RC, Snyder PJ, Carrillo MC, Thies B, Phelps CH: The diagnosis of mild cognitive impairment due to Alzheimer's disease: recommendations from the National Institute on Aging-Alzheimer's Association workgroups on diagnostic guidelines for Alzheimer's disease. Alzheimers Dement 2011;7:270-279.

24 Jorm AF: The Informant Questionnaire on Cognitive Decline in the Elderly (IQCODE): a review. Int Psychogeriatr 2004;16:275-293.

25 American Psychiatric Association: Diagnostic and Statistical Manual of Mental Disorders, ed 4, text rev. Washington, American Psychiatric Association, 2000.

26 Morris JC: The Clinical Dementia Rating (CDR): current version and scoring rules. Neurology 1993;43:24122414.

27 Copeland JR, Dewey ME, Griffiths-Jones HM: A computerized psychiatric diagnostic system and case nomenclature for elderly subjects: GMS and AGECAT. Psychol Med 1986;16:89-99.

28 Prince M, Acosta D, Chiu H, Copeland J, Dewey M, Scazufca M, Varghese M: Effects of education and culture on the validity of the Geriatric Mental State and its AGECAT algorithm. Br J Psychiatry 2004;185:429-436.

29 Prince M: Methodological issues for population-based research into dementia in developing countries. A position paper from the 10/66 Dementia Research Group. Int J Geriatr Psychiatry 2000;15:21-30.

30 Prince M, Ferri CP, Acosta D, Albanese E, Arizaga R, Dewey M, Gavrilova SI, Guerra M, Huang Y, Jacob KS, Krishnamoorthy ES, McKeigue P, Rodriguez JL, Salas A, Sosa AL, Sousa RM, Stewart R, Uwakwe R: The protocols for the 10/66 Dementia Research Group population-based research programme. BMC Public Health 2007; 7:165.

31 Persson K, Brækhus A, Selbæk G, Kirkevold Ø, Engedal K: Burden of care and patient's neuropsychiatric symptoms influence carer's evaluation of cognitive impairment. Dement Geriatr Cogn Disord 2015;40:256267. 